\title{
Quantitative assessment of tricuspid regurgitation using pulsed Doppler echocardiography
}

\author{
B DIEBOLD, R TOUATI, D BLANCHARD, G COLONNA, J L GUERMONPREZ, \\ P PERONNEAU, J FORMAN, P MAURICE \\ From the Cardiology Clinic and INSERM Unit 256, Hôpital Broussais, Paris, France
}

SUMMARY Tricuspid valve regurgitation was assessed quantitatively by measuring blood flow velocity in the vena cava using a pulsed Doppler velocimeter. A non-invasive index of regurgitation was obtained by calculating the ratio between the maximum amplitudes of the systolic and diastolic components of the velocity curves. The index was compared with the angiographic grading of regurgitation in 70 patients after right heart catheterisation; the results were closely correlated. Using the Doppler index the differences between the groups defined according to their angiographic grade were significant.

Thus measurement of blood flow velocity in the vena cava appears to quantify accurately the severity of tricuspid regurgitation.

The evaluation of tricuspid regurgitation is frequently difficult: clinical examination, ${ }^{1}$ jugulogram, ${ }^{2}$ jugular vein flow measurement, ${ }^{3}$ and echocardiogram ${ }^{4}$ give reliable information only in the patients with major leaks. Contrast echocardiography of the inferior vena cava has recently been proposed ${ }^{5}$; unfortunately, it is neither specific nor quantitative. As exploration during surgery is unreliable right heart catheterisationincluding selective right ventricular angiographyhas until now been essential. ${ }^{6}$ Since tricuspid regurgitation rarely appears in isolation, right ventricular angiography must be performed at the end of the invasive procedure; this, however, increases the risks attached to the procedure. For this reason, tricuspid lesions are often overlooked. This supports the need for reliable non-invasive diagnosis of these lesions. Recently, the non-invasive examination of the right atrium using Doppler echocardiography has been proposed for the diagnosis ${ }^{7}$ and semiquantification ${ }^{9}$ of tricuspid incompetence. The aim of this study was to examine the possibility of quantitively assessing tricuspid regurgitation by analysing the blood flow pattern in the inferior vena cava.

\section{Patients and methods}

\section{PATIENT SELECTION}

Seventy patients admitted to hospital for the invasive investigation of heart disease were studied. They Work supported by a grant from CNAMTS.

Accepted for publication 22 June 1983 underwent right heart catheterisation with right ventricular angiography followed by Doppler echocardiography up to three days later. There were 39 women and 31 men (mean age 36 years, range 15-69). Of these, 37 patients had mitral valve disease, 21 aortic and mitral lesions, one aortic insufficiency, five a prosthetic mitral valve (including one patient with three), one an atrioventricular canal defect and aortic regurgitation, one corrected transposition and infundibular pulmonary stenosis, one Ebstein's anomaly, one constrictive pericarditis, one cardiomyopathy, and one isolated tricuspid regurgitation.

\section{CATHETERISATION}

All patients underwent right heart catheterisation including selective right ventricular angiography. The latter was performed using a catheter positioned with its end close to the apex of the right ventricle; the infusion rate of contrast material was $20 \mathrm{ml} / \mathrm{s}$ during $3 \mathrm{~s}$; cineangiography was performed at 40 frames/s. The degree of regurgitation was graded by two independent observers according to a five-grade semiquantification: grade 0 , no regurgitation of contrast into the right atrium; grade 1 , minimal flush of contrast into the right atrium; grade 2, major leak but not resulting in total opacification of the right atrium; grade 3 , leak resulting in total opacification of the right atrium but only after several systoles; and grade 4 , regurgitation producing total opacification of the right atrium on the first systole after the start of the contrast infusion. 


\section{DOPPLER EXAMINATION}

The principle of pulsed Doppler blood velocity measurement and the main features of the prototype used in this study have been described ${ }^{10-12}$ and are therefore briefly summarised.

Ultrasonic waves are emitted during an adjustable period of 1-4 $\mu$ s with a pulse repetition rate of 5 or $10 \mathrm{KHz}$ and an emission frequency of $4 \mathrm{MHz}$; the ultrasound reflected by structures or backscattered by blood cells is analysed for imaging and Doppler detection; the imaging system operates during the whole period separating the pulsed bursts; it gives an M-mode image corresponding to the axis of the analysis and covering the maximum analysable depth; the Doppler circuit operates only after a delay and during a period which can be selected, so that depth and width of the sample volume can be chosen. The measurement is performed using the Doppler effect: the frequency of ultrasounds backscattered by the moving target is slightly different with respect to the emitted frequency $(F)$. This shift depends on $F$, on the ultrasound propagation speed in tissues, on the velocity of the target, and on the cosine of the angle between the axis of propagation of ultrasound and the axis of target displacement. Consequently, Doppler measurement is not possible if ultrasonic beam and flow are perpendicular, and no quantification is possible without the angle factor being known or eliminated.

The Doppler signal consists of several frequencies that may be displayed using a spectrum analyser. An instantaneous mean velocity curve was used in this study. It was obtained by using a zero crossing counter; its accuracy is ensured, provided that the signal-to-noise ratio is high and that a high pass filter is used to remove low frequencies due to wall motion.

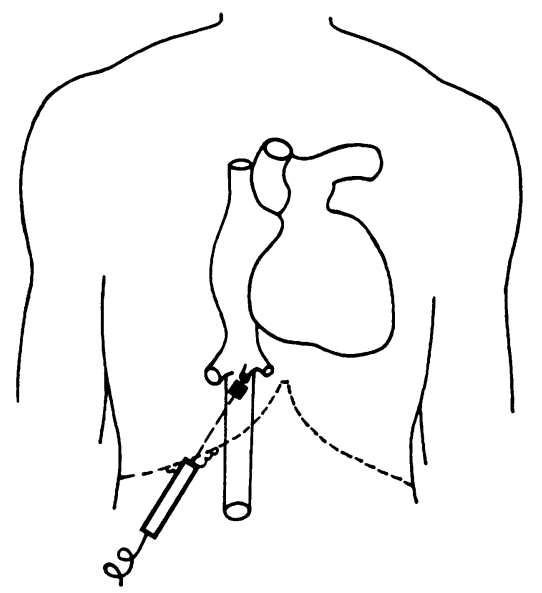

Fig. 1 Position of the transducer for recording the velocity curve of the blood flow in the inferior vena cava.
The analogue velocity curve and the electrocardiogram were monitored either with a two channel recorder or, together with the M-mode echo, with a fibreoptic stripchart recorder (LS8 Honeywell). During the last part of the study we used a pulsed Doppler velocimeter combined with a cross sectional imager.

The patient was placed in the supine position. The transducer was located on the epigastric area or on one of the lower right intercostal spaces to display the inferior vena cava behind the liver (Fig. 1). The blood flow in the vena cava was regulated by the heart and by respiration. In about half the patients it was not possible to obtain a suitable signal within the vena cava; the flow curves were therefore recorded from the end of one of the venae hepaticae, since they lie slightly more superficially and at a more favourable angle (Fig. 2) and their flow patterns have the same characteristics as those of the vena cava.

\section{Velocity curve patterns}

The curves obtained during Doppler echocardiography reflect the time dependent variations of the average velocity encountered within the sample volume. They display blood flow approaching the transducer as positive and flow running away as negative. The curves were recorded during expiratory apnoea.

Normal flow-This was studied in 10 healthy subjects (Fig. 3). The curves were consistent with previously reported patterns ${ }^{13}{ }^{14}$ : there was a predominant negative systolic component (s), the diastolic component (d) had a smaller amplitude, it ended at the onset of the atrial contraction which may produce a brief flow reversal. In the patient with atrial fibrillation the relative amplitude of the systolic component was

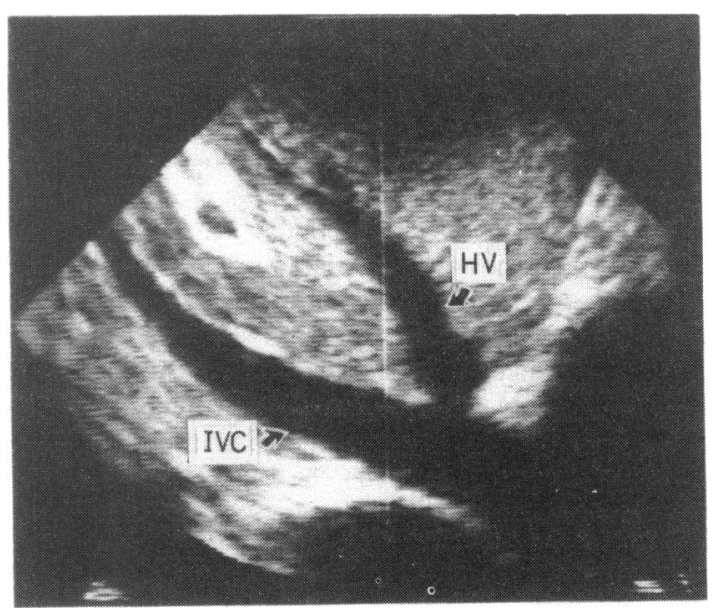

Fig. 2 Cross sectional imaging during a Doppler examination of the inferior vena cava (IVC). The vena hepatica (HV) lies more superficially at a more favourable angle in relation to the ultrasonic beam. 

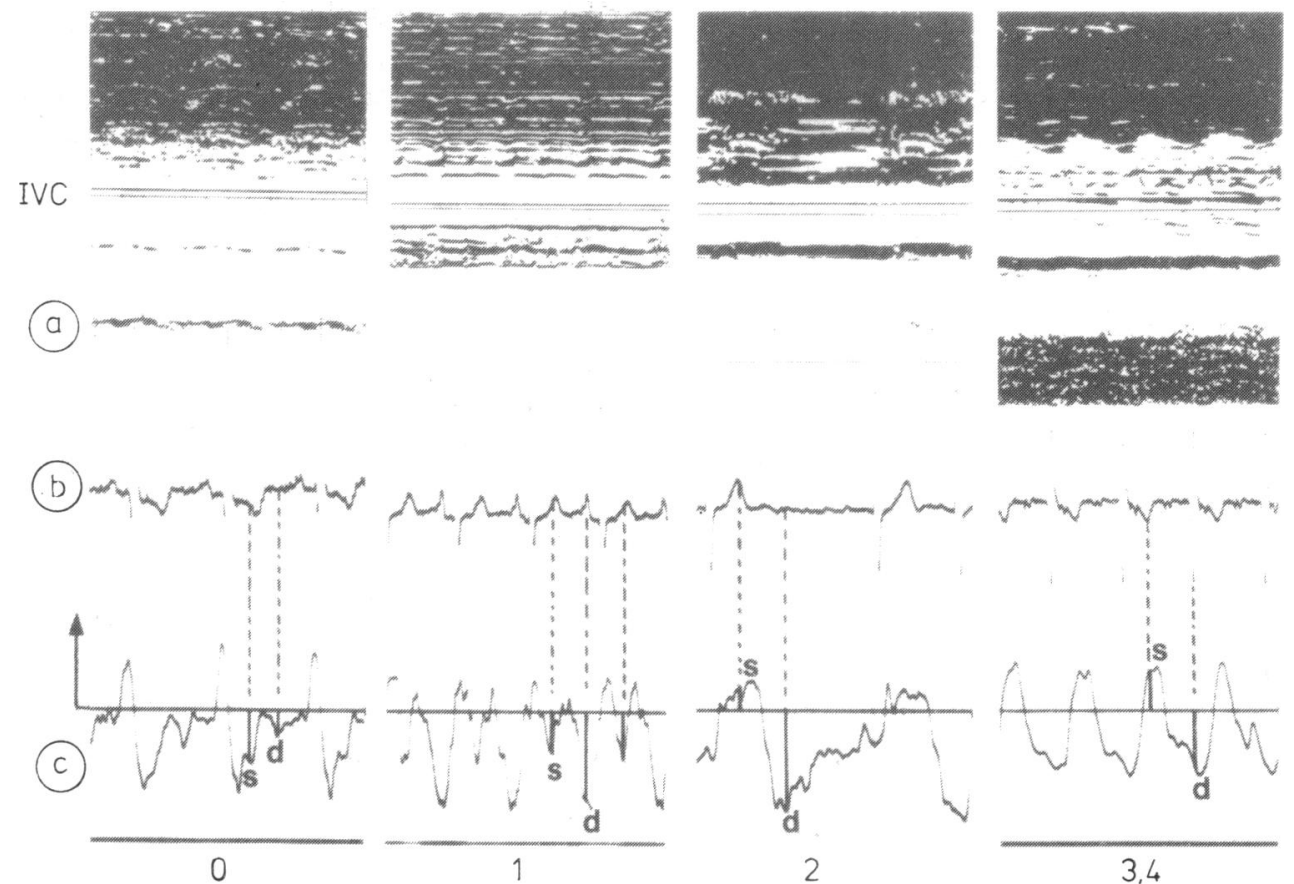

Fig. 3 (a) Simultaneous $M$-mode echo, (b) electrocardiogram, and (c) velocity curve showing variations according to the severity of tricuspid regurgitation. Grade 0 , no regurgitation; 1 , minimal regurgitation; 2 , moderate regurgitation; 3, severe regurgitation; 4, massive regurgitation: s, systolic component; $d$, diastolic component; IVC, inferior vena cava.

smaller and the end-diastolic flow reversal disappeared (Fig. 4). It should be noted that since pressure variations are heart induced the curves obtained from

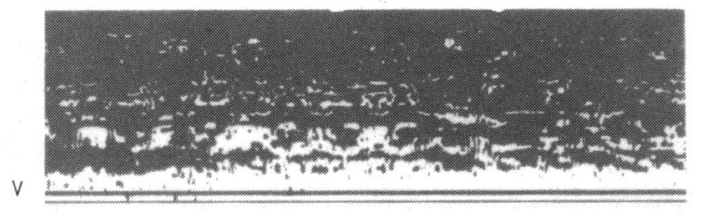

(a).
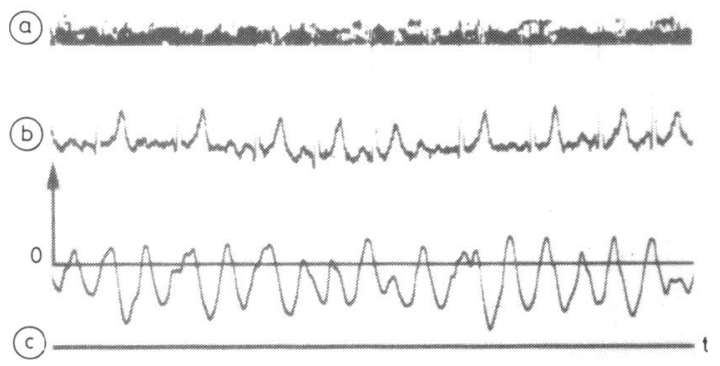

Fig. 4 (a) Simultaneous $M$-mode echo, (b) electrocardiogram, and $(c)$ velocity curve in a patient with atrial fibrillation and without tricuspid regurgitation. $O$, zero velocity; $t$, time; arrow indicates direction of retrograde flow. the vena cava or venae hepaticae lag in relation to the events of the cardiac cycle owing to the venous compliance; nevertheless, flow patterns are not modified by the position of the sample volume as long as recordings are not performed at the level of the distal branches of the venae hepaticae.

Tricuspid regurgitation-In the patient with mild regurgitation, the amplitude of the systolic component decreased (Fig. 3, grade 1); if the regurgitation is moderate or severe the normal systolic anterograde component is replaced by a positive systolic retrograde component (Fig. 3, grades 2-4), as has already been shown during thoracotomy ${ }^{15}$ or when a catheter tip velocimeter is used. ${ }^{16}$

Index of regurgitation-Since recordings were performed at some distance from the heart and at some distance from upstream vessel curvature we assumed, firstly, that the general shape of the velocity profile does not vary much during the cardiac cycle and thus that a recording performed more or less close to the centre of the vessel is representative along the cycle; and secondly, that systolic and diastolic flows are at the same angle in relation to the ultrasonic beam. Under these conditions, the calculation of a ratio between two components of the velocity curve gives an 
(a)

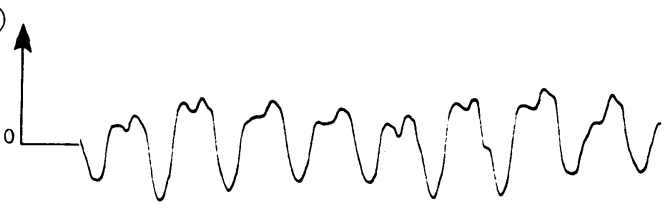

(b)

Fig. 5 Velocity curve and simultaneous electrocardiogram in a patient with impaired compliance of the right ventricle. $O$, zero velocity; arrow indicates direction of retrograde flow.

index that does not depend on the angle and is representative of velocity variations. Furthermore, since the diameter modifications of the vena cava during the cardiac cycle are small the index of velocity variations can be considered to reflect the variations in the flow volume in the vena cava. This type of ratio can be calculated using either the velocity amplitudes or the area between the baseline and the different compo-

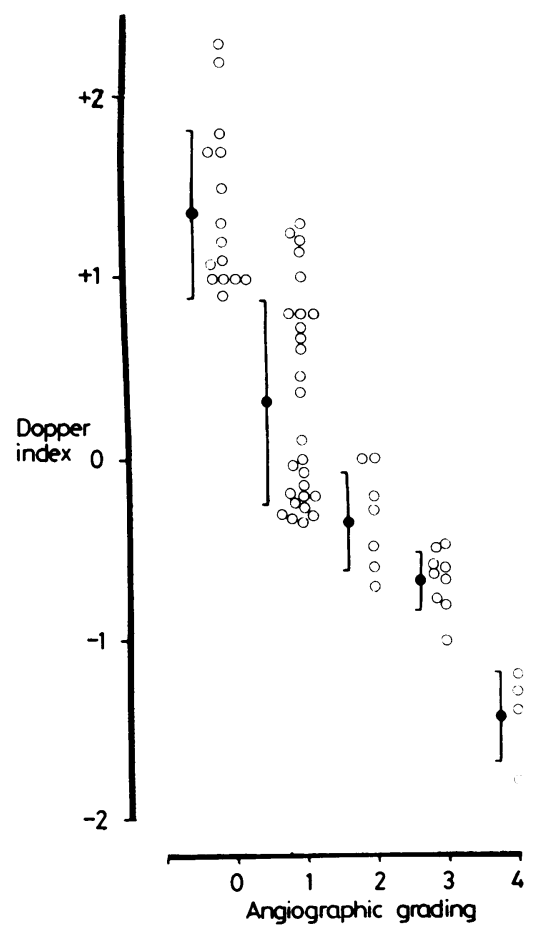

Fig. 6 Comparison of results between the Doppler index and the angiographic grade of tricuspid regurgitation. Bars represent means $\pm S D ; n=62$. nents of the curve. Sometimes in patients with sinus rhythm or with decreased right ventricular compliance the end-diastolic reversal component (Fig. 5) may fuse with a systolic reversal flow, preventing any clear delineation of the area related to the latter. We therefore calculated the ratio of the amplitude measured at the end of the $T$ wave of the electrocardiogram to the peak diastolic anterograde amplitude.

\section{Results}

The interobserver and intraobserver reproducibility of the curves was evaluated in 12 patients by two different observers. Fig. 6 shows the results with the Doppler technique and angiography.

\section{DIAGNOSIS}

When all the patients are considered the sensitivity and the specificity depended on the value of the Doppler index selected as the lower limit of normal: with a limit at 1.3 the sensitivity was $100 \%$ and the specificity $40 \%$; with a limit at 0.8 the sensitivity was $89 \%$ and the specificity $100 \%$. These results are due to the overlap between patients with grade 0 and grade 1 regurgitations. If only moderate to severe regurgitations (grades 2-4) are considered the sensitivity and the specificity were $100 \%$.

\section{QUANTIFICATION}

Using analysis of variance the Doppler index showed a highly significant difference between the groups defined according to the angiographic grade $(\mathrm{F}=47.84 ; \mathrm{p}<0.0001$ ) (Table). On the one hand, there were significant overlaps between the consecutive grades (grades 0 and 1 , grades 1 and 2, etc); on the other hand, there was no overlap between grades 0 and 2 , grades 1 and 3 , and grades 2 and 4 ; the mean value of the Doppler index increased progressively with increasing severity of the tricuspid regurgitation (Table). There was a correlation coefficient of $r=0.90$ between the angiographic grade and the Doppler index.

Table Value of the Doppler index in relation to angiographic grading of the severity of tricuspid regurgitation

\begin{tabular}{lrl}
\hline Angiographic grade & Mean & $S D$ \\
\hline 0 & 1.39 & \pm 0.44 \\
1 & 0.32 & \pm 0.55 \\
2 & -0.36 & \pm 0.26 \\
3 & -0.68 & \pm 0.16 \\
4 & -1.43 & \pm 0.22 \\
\hline
\end{tabular}

Significance of difference (analysis of variance): between groups 0 and $1, p<0.001$; groups 1 and $2, p<0.001$; groups 2 and $3, p<0.005$; groups 3 and $4, p<0.001$. 


\section{Discussion}

\section{FLOWS}

Flows within the venae cavae are generated by pressure gradients depending on the mechanical activity of the heart. ${ }^{1718}$ In normal subjects the sum of the effects of atrial relaxation and of ventricular systole (with the lowering of the ventricular floor) generates a systolic flow towards the right atrium. A second component with the same orientation occurs during the first part of diastole, corresponding to the passive filling of the right ventricle. During atrial contraction the lack of a valvular system between right atrium and venae cavae leads to a reversal of flow in the latter.

In tricuspid regurgitation the valvular leak allows the onset of a systolic jet within the right atrium; it may be detected as a murmur on intracardiac phonocardiography. ${ }^{19}$ This jet generates a pressure wave which itself produces a flow wave. The amount of regurgitant blood is the product of the mean jet velocity multiplied by the size of the orifice through which the regurgitation occurs; furthermore, thin jets with high velocities-corresponding to moderate regurgitation-may propagate far within right atrium. The pressure wave accounts for the systolic expansion of jugular veins and of the liver and for the systolic flow reversal generated in place of the normal anterograde one within the venae cavae.

In atrial fibrillation the lack of atrial contraction leads to a decrease in the systolic anterograde component. There is, however, still controversy over whether or not atrial fibrillation may induce mild tricuspid regurgitation. ${ }^{20}$

\section{M-MODE ECHOCARDIOGRAPHY}

Most of the patients in this study were examined using $M$-mode echocardiographic imaging. A few were examined using a duplex unit combining the pulsed Doppler velocimeter with a mechanical sector scanner. This technology has not radically modified either the technique or the results: it both aids localisation of the inferior vena cava and reduces the quality of the Doppler signal (signal to noise ratio).

Spectrum analysers have been proposed for processing the Doppler signal. They display the different components of the signal. In practice, they allow easier pattern recognition but until now have not provided more accurate mean velocity curves than zero crossing counters.

\section{PATIENT EXAMINATION}

The recording of a systolic jet behind the tricuspid valve $^{7}$ has been suggested as a method of diagnosing tricuspid regurgitation: it has proved to be reliable in several studies. An analysis of the propagation of the jet within the right atrium has been proposed to

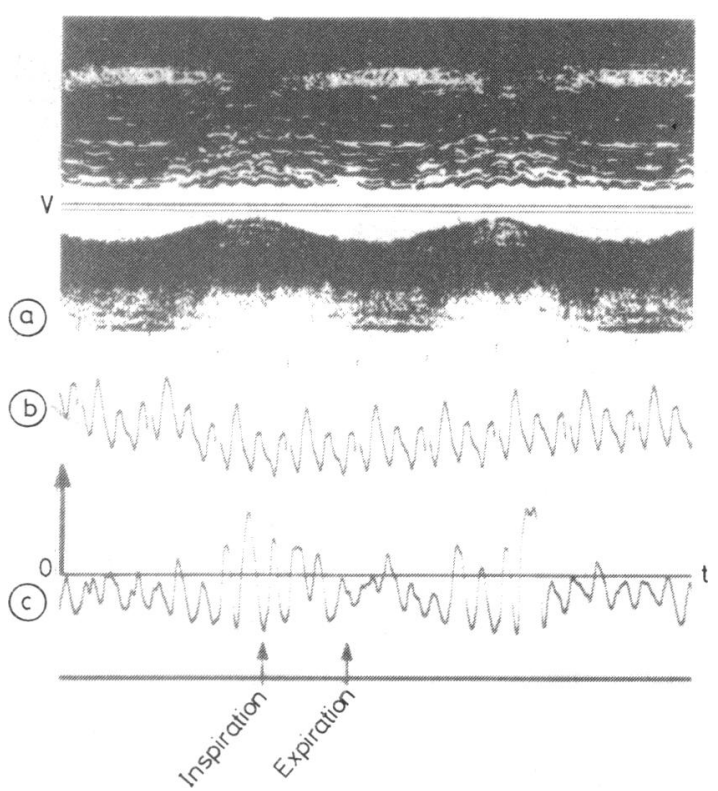

Fig. 7 (a) Simultaneous $M$-mode echo, (b) electrocardiogram, and $(c)$ velocity curve showing the respiratory variations and modifications due to atrial flutter. $O$, zero velocity; $t$, time; $V$, position of velocity sample volume.

evaluate the severity of the leak. ${ }^{8}$ The results indicated an important overlap between the groups, probably because of the nature of the flows at the level of the right atrium. Information obtained by this means clearly depends on the shape of the jet. The calculation of a ratio at the level of the tricuspid orifice ${ }^{21}$ does

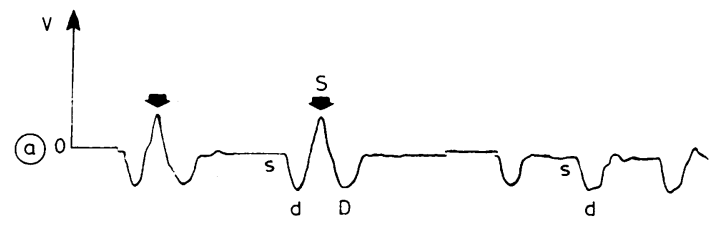

(b)

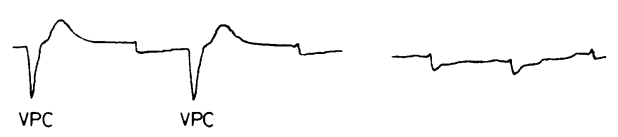

Fig. 8 (a) Velocity curve and (b) simultaneous electrocardiogram showing modifications induced by ventricular premature contractions (VPC): the left side shows recording with $V P C$, the right side recording without rhythm abnormality: s and $d$, components related to the sinus rhythm; $S$ and $D$, components corresponding to VPC. 


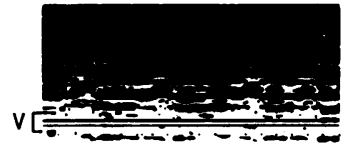

(a)
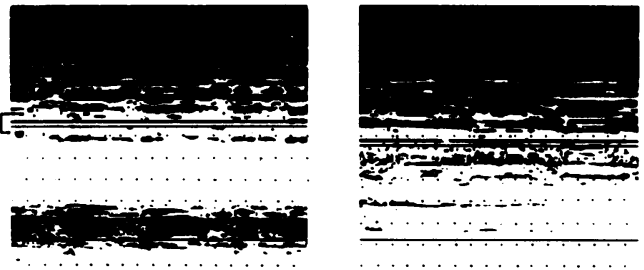

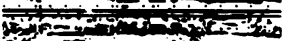
$\therefore$

(b)
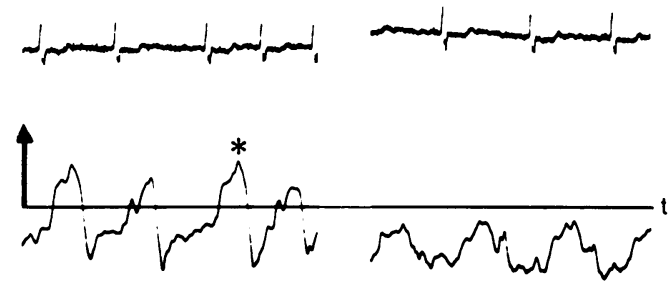

(C)

IVC

PV

Fig. 9 (a) Simultaneous M-mode echo, (b) electrocardiogram, and (c) velocity curve in severe tricuspid regurgitation: the left side shows the flow pattern in the inferior vena cava (IVC), the right side the flow pattern in the portal vein $(P V) ; V$, position of velocity sample volume. ${ }^{\star}$ Indicates the systolic flow reversal.

not take into account the angle factor and the variation in the cross-section of the flows during the cardiac cycle. The investigation of venae cavae has already been proposed either peroperatively ${ }^{15}$ or by a non-invasive technique. ${ }^{8}$ These date are consistent with our results.

In practice some technical points need to be emphasised.

(1) As the vena caval velocities and the amount of regurgitant blood vary with intrathoracic pressurein particular with respiration (Fig. 7)-it is necessary to carry out the examination during open glottis apnoea; this condition is more easily achieved at the end of expiration.

(2) Some rhythm or conduction abnormalities may be misleading, although they were not encountered during our study. Atrial flutter may produce important modifications of vena caval blood flow (Fig. 7); in atrioventricular block each atrial contraction may produce a flow reversal; premature ventricular contraction may produce a systolic component reversal (Fig. 8); sometimes in patients with pacemakers cardiac contractions due to pacing may produce a flow reversal that is otherwise missing. When velocity curves are recorded at the level of the vena hepatica, in cases of major tricuspid regurgitation, the portal flow may be confusing: it is blocked by the systolic pressure wave which makes the liver pulsate (Fig. 9).

(3) A poor setting of the high pass filters may also produce misleading results. These filters are necessary to avoid artefacts due to wall motion. Unfortunately, they remove the low frequencies related to slowly moving blood cells. If the cutoff frequency of the filters is too high a small reversal component may be cancelled.

(4) The size of the sample volume prevents the risk of measuring a non-representative juxtaparietal velocity component.

\section{RESULTS}

The sensitivity and the specificity of the technique depend on the threshold ratio selected. In our experience, a threshold of 0.8 gives a specificity of $100 \%$; recently, a study of a smaller number of patients proposed a threshold of 0.3 . This difference is probably due to the well known limitations of the gold standard. Nevertheless, in practice the method appears to be highly specific. From a theoretical point of view, Ebstein's anomaly could lead to false positive results owing to the systolic contraction of the intermediate chamber; fortunately, it is usually associated with severe tricuspid incompetence.

For the quantitative evaluation of tricuspid leak the correlation coefficient appears to be interesting. Despite the overlap between the groups because of the limitations of right ventricular angiography, our results were appreciably better than those reported with other non-invasive methods. This should not, however, disguise the fact that the same leak can be propagated differently in the vena cava in each patient according to the compliance of the venous system. In terms of quantification, the only comparable study has been carried out during surgery ${ }^{15}$; it showed comparable results at the level of the superior vena cava and using open chest measurement.

\section{CONCLUSION}

Doppler echocardiography allows recordings of blood flow velocity curves at the level of the inferior vena cava. The calculation of a ratio between systolic and diastolic components of these curves gives an index for quantifying tricuspid regurgitation. When compared with those of catheterisation these results are unequalled by any other non-invasive technique.

\section{References}

1 Sepulveda G, Lukas DS. The diagnosis of tricuspid $\mathrm{N}$ insufficiency: clinical features in 60 cases with associated mitral valve disease. Circulation 1955; 11: 552-63.

2 Mueller $\mathrm{O}$, Shillingford J. Tricuspid incompetence. $\mathrm{Br}$ Heart f 1954; 16: 195-206.

3 Kalmanson D, Veyrat C, Chiche P. Diagnostic par voie externe des cardiopathies droites et des shunts intracardiaques gauche-droit à l'aide du fluxmètre directionnel à effet Doppler. Presse Medicale 1970; 78: 1053-6.

4 Feigenbaum H. Acquired valvular heart disease. In:

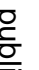


Echocardiography. 3rd ed. Philadelphia: Lea and Febiger, 1981: 286-8.

5 Meltzer RSV, Hoogenhuyze D, Serruys PW, Haalebos MMP, Hugelholtz PG, Roelandt J. Diagnosis of tricuspid regurgitation by contrast echocardiography. Circulation 1981; 63: 1093-9.

6 Cairns KB, Kloster FE, Bristow JD, Lees MH, Griswold HE. Problems in the hemodynamic diagnosis of tricuspid insufficiency. Am Heart $\mathcal{f}$ 1968; 75: 173-9.

7 Waggoner AD, Quinones MA, Young JB, et al. Pulsed Doppler echocardiographic: detection of right-sided valve regurgitation experimental results and clinical significance. Am f Cardiol 1981; 47: 279-86.

8 Garcia-Dorado D, Falzgraf S, Almazan A, Delcan JL, Lopez-Bescos L, Menarguez L. Diagnosis of functional tricuspid insufficiency by pulsed-wave Doppler ultrasound. Circulation 1982; 66: 1315-21.

9 Miyatake K, Okamoto M, Kinoshita N, et al. Evaluation of tricuspid regurgitation by pulsed Doppler and two dimensional echocardiography. Circulation 1982; 66: 777-84.

10 Diebold B, Theroux P, Bourassa MG, et al. Noninvasive pulsed Doppler study of mitral stenosis and mitral regurgitation: preliminary study. $B r$ Heart $\mathcal{f} 1979$; 42: $168-75$.

11 Blanchard D, Diebold B, Peronneau P, et al. Noninvasive diagnosis of mitral regurgitation by Doppler echocardiography. Br Heart f 1981; 45: 589-93.

12 Diebold B, Peronneau P, Blanchard D, et al. Noninvasive quantification of aortic regurgitation by Doppler echocardiography. Br Heart $\mathcal{f}$ 1983; 49: 167-73.

13 Froysaker $T$. Normal flow pattern in the superior vena cava in man during thoracotomy. Scand $\mathcal{F}$ Thorac Cardiovasc Surg 1972; 6: 22-32.
14 Wexler L, Bergel DH, Gabe IT, Makin GS, Mills CJ. Velocity of blood flow in normal human venae cavae. Circ Res 1968; 23: 349-59.

15 Froysaker T. Anomalies of the superior vena caval flow pattern in patients with tricuspid insufficiency. Scand $\mathcal{F}$ Thorac Cardiovasc Surg 1972; 6: 234-46.

16 Nolan SP, Botero LM, Inocencio EC, Rawitscher RE, Lee RJ. Detection of tricuspid valvular regurgitation with a catheter velocitometer. Circulation 1971; 43-44 (suppl 1): 57-61.

17 Morgan BC, Abel FL, Mullins GL, Guntheroth WG. Flow patterns in cavae, pulmonary artery, pulmonary vein, and aorta in intact dogs. Am $\mathcal{F}$ Physiol 1966; 210: 903-9.

18 Pinkerson AL, Luria MH, Freis ED. Effect of cardiac rhythm on vena caval blood flows. Am $\mathcal{F}$ Physiol 1966; 210: 505-8.

19 Se Do Cha, Gooch AS, Maranhao V. Intracardiac phonocardiography in tricuspid regurgitation: relation to clinical and angiographic findings. Am $\mathcal{F}$ Cardiol 1981; 48: 578-83.

20 Kalmanson D, Veyrat C, Chiche P. Venous return disturbances induced by arrhythmias. A transcutaneous, instantaneous flowmetric study at the site of the jugular vein. Cardiovasc Res 1970; 4: 279-90.

21 Veyrat C, Kalmanson D, Farjon M, Manin JP, Abitbol G. Non-invasive diagnosis and assessment of tricuspid regurgitation and stenosis using one and two dimensional echo-pulsed Doppler. Br Heart $\mathcal{F}$ 1982: 47: 596-605.

Requests for reprints to Professor J L Guermonprez, Clinique Cardiologique, Hôpital Broussais, 96 Rue Didot, 75014 Paris, France. 